\title{
Zur Notwendigkeit und Chance einer sozial(räumlich)en Perspektive und einer Einmischung Sozialer Arbeit im interdisziplinären Diskurs zum Wohnen
}

\author{
Sylvia Beck
}

Eingegangen: 28. Mai 2021 / Angenommen: 20. Oktober 2021 / Online publiziert: 19. Januar 2022 (C) Der/die Autor(en) 2022

Zusammenfassung Das Thema Wohnen wird derzeit angesichts von Wohnungsmarktdynamiken vielfach diskutiert, bleibt dabei jedoch weitgehend auf Fragen einer bezahlbaren Wohnraumversorgung fokussiert. Exemplarisch aufgezeigte aktuelle Entwicklungen verweisen jedoch zugleich auf gesellschaftliche und individuelle Anforderungen ans Wohnen, die Transformationsprozesse mitbedingen. Darin werden auch Selbstzuständigkeiten wie auch deren Vereinnahmung sichtbar, die hinsichtlich neuerlicher Ausschlüsse kritisch zu hinterfragen sind. Gestützt auf das Konzept von „Wohnen als sozial(räumlich)e Praxis“ plädiert der Beitrag für eine sozialräumliche und lebensweltorientierte Sicht auf Wohnen im Kontext Sozialen Wandels. Er macht sich damit zugleich für eine Einmischung Sozialer Arbeit im interdisziplinären Diskurs zum Wohnen und in Wohn- und Stadtentwicklungen stark, um für ermöglichende, sozial gerechte Strukturen einzutreten, aber auch Aneignungsprozesse in ihrer Relevanz herauszustellen.

Schlüsselwörter Soziales Wohnen · Wohnforschung · Sozialraum · Lebensweltorientierung · Einmischung 


\title{
On the need and opportunity of a socio-spatial perspective and involvement of social work in the interdisciplinary discourse on housing
}

\begin{abstract}
The issue of housing is currently widely discussed in the light of housing market dynamics, but remains largely focused on issues of affordable housing supply. However, examples of current developments point to social and individual requirements in housing that involve transformation processes. This also reveals selfresponsibility as well as their capture/the utilization thereof, which must be critically questioned with regard to new exclusions. Based on the concept of "housing as a socio-spatial practice", this article advocates a socio-spatial and life-oriented view of housing in the context of social change. At the same time, it advocates for involvement of social work in the interdisciplinary discourse on housing and in residential and urban development, in order to advocate for enabling, socially just structures, but also to highlight the relevance of appropriation processes.
\end{abstract}

Keywords Social housing $\cdot$ Residential research $\cdot$ Social space $\cdot$ Life world orientation · Involvement

Das Wohnen ist in den letzten Jahren zu einem bedeutenden, gesellschaftlichen wie politischen Thema geworden und steht im Zentrum vielfältiger Debatten und Analysen. Diskutiert werden dabei vornehmlich steigende Wohnungspreise resp. Wohnungsmieten und damit einhergehende wachsende Wohnungsnöte wie auch Verdrängungsprozesse (Stichwort Gentrifizierung) sowie notwendige Gegenmaßnahmen hierfür. Einvernehmlich ist bereits von einer neuerlichen Wohnungsfrage die Rede, womit im Diskurs auf die dramatische Wohnsituation im Zuge der Industrialisierung verwiesen wird (Schönig 2013; Holm 2014; Beck und Reutlinger 2019). Während die historische Wohnungsfrage jedoch - zumindest rückwirkend - im Verweis auf gesellschaftliche Umbrüche, auf die darin gründende „Soziale Frage“ und deren Lösung durch umfassende soziale Reformen analysiert wurde/wird, findet eine solche grundlegendere gesellschaftliche Rahmung der aktuellen Wohnentwicklungen bislang nur punktuell statt. Es bleibt m. E. unzureichend im Blick, wie vielfältig und worüber sich Veränderungen im Wohnen heute bedingen, also was Schubkräfte und Motoren von derzeitigen Entwicklungen sind, aber auch welche Implikationen damit für eine soziale Gestaltung von Wohnverhältnissen einhergehen. Wohndebatten sind nur selten im gesellschaftlichen Bedingungsgefüge und darin liegenden individuellen Herausforderungen gerahmt - also wie sich Wohnen als Handlungszusammenhang über gesellschaftliche Verhältnisse mitbedingt und welche neuen, auch unterschiedlichen Anforderungen sich für den*die Einzelne*n daraus ergeben. Wenig thematisiert bleibt auch eine Perspektive darauf, wie Wohnende selbst ihr Wohnen erfahren, und wie sie allenfalls in notwendig gewordener selbstverantwortlicher Lebensführung auch als Mitakteur*innen in diesen Wohnentwicklungen zu betrachten sind oder darin gerade neue Ausschlüsse erfahren. Dies sind Indizien, Wohnen nicht nur als Frage der Wohnraumversorgung, sondern notwendig auch stärker von einer sozialen Seite - nämlich gerahmt von gesellschaftlichen Entwicklungen und mit Blick auf heutige alltägliche Lebensführungen -, aber auch als Prozess zu the- 
matisieren, der sich vielseitig bedingt. Es sind dies zugleich Indizien, die m.E. die Soziale Arbeit, die immer auch schon in Wohnverhältnisse involviert ist, vor allem in ihrer kritischen Haltung - als Gestalterin des Sozialen - viel stärker auf den Plan rufen müsste als bislang. Dieser Beitrag intendiert deshalb, die bisherige Thematisierung aktueller Wohnentwicklungen in ihrer sozialen Dimension zu stärken und damit Transformationsprozesse im Wohnen umfassender und letztlich auch in ihrem Ermöglichungspotenzial zu betrachten. Zugleich geht es darum, die Notwendigkeit und Chance eines Beitrags Sozialer Arbeit im interdisziplinären Wohndiskurs wie auch in der konkreten Gestaltung von Wohnverhältnissen herauszuarbeiten und zur Diskussion zu stellen.

Hierfür werde ich im Folgenden vorweg eher blitzlichtartig aktuelle Verhandlungen des Wohnens aufzeigen, vor deren Hintergrund ich mich für eine ganzheitliche Sicht ausspreche, welche Wohnen heute im Kontext Sozialen Wandels, aber auch mit darin liegenden individuellen Bewältigungsherausforderungen rahmt und zugleich strukturelle sowie räumliche Gegebenheiten des Wohnens - also die konkrete Wohnraumversorgung, aber auch deren Einbettung in Stadt-/Raumentwicklung mitberücksichtigt bzw. dazu in Beziehung setzt. Diese Überlegungen basieren in ihrem Kern auf dem Konzept „Wohnen als sozialräumliche Praxis“ (Beck 2021), das im Zuge einer empirischen Arbeit zu Gemeinschaftlichen Wohnprojekten gewonnen werden konnte, und hier für eine systematische Betrachtung von Wohnentwicklungen dienen soll. Raumtheoretisch fundiert, eröffnet dies eine analytische Perspektive darauf, wie Wohnen im gesellschaftlichen Kontext im Mitwirken vieler Akteur*innen beständig sozial, strukturell und räumlich hergestellt resp. bewahrt oder verändert wird. Dies bildet zugleich die Basis, um anschließend zu verdeutlichen, dass und wie Soziale Arbeit in der diskursiven Verhandlung des Wohnens, aber - im Sinne von Einmischung - auch in der praktischen, interdisziplinären Gestaltung von Wohnverhältnissen notwendig und chancenreich beitragen kann. Was nicht zuletzt weitere empirische Zugänge, aber auch eine Rollenklärung ihrerseits erfordert.

\section{Aktuelle (praktische und diskursive) Verhandlungen des Wohnens}

Im Mittelpunkt aktueller Wohndiskussionen stehen meist problematische Wohnentwicklungen, wie sie insbesondere seit der Finanzkrise 2008 und noch massiver in den letzten Jahren wahrzunehmen sind. Sichtbar werden ein zugespitzter Wohnungsmarkt, wachsende Wohnungsnöte und Armutslagen sowie Verdrängungsprozesse - und zwar entlang bisheriger Ungleichverhältnisse, aber auch für neue Gruppen (der so bezeichneten gesellschaftlichen Mitte). Als Ursachenzusammenhänge werden vor allem ein Abbau sozialstaatlicher Regulierungen, neoliberale Umstrukturierungsprozesse und wachsende Einflüsse des Finanzmarktes auf dem Wohnungsmarkt analysiert (Schönig 2018). Konkret wird etwa die Vernachlässigung des Sozialen Wohnungsbaus seit den 1980er Jahren kritisiert, was zu einem auch jetzt 
noch weiter schrumpfenden Bestand an Sozialwohnungen führt. ${ }^{1}$ Auch die Abschaffung der Gemeinnützigkeit in der Wohnraumversorgung im Jahr 1990, die nicht nur den Verlust einer vielfältigeren sozialen Trägerlandschaft im Wohnen, sondern auch sozial gestalteten Wohnraums zur Folge hat (Holm 2018), wird kritisch eingeordnet. Ebenso die Verlagerung von Zuständigkeiten vom Bund auf die Länder und (finanzschwache) Kommunen im Zuge der Förderalismusreform 2006. Medial am präsentesten wirkt, wie ehemals kommunales Wohneigentum privatisiert und damit seiner sozialen Verwertung entzogen wurde - wie am Beispiel der zwischenzeitlich börsennotierten Deutsche Wohnen ersichtlich wird, die alsbald in Vonovia als dann größtem europäischem Wohnungsunternehmen aufzugehen scheint. Auch wird insgesamt eine Finanzialisierung - also eine Vermarktlichung und Ökonomisierung im Wohnsektor kritisiert (Heeg 2018). Diese vollzieht sich nicht nur durch ein immer stärkeres Agieren national und global tätiger Finanzakteur*innen, darunter auch eine wachsende Zahl an Versicherungen, welche (deutsche) Wohnimmobilien als sicheren (Anlage-)Hafen nutzen. Hingegen sind es auch viele private Kleinanleger*innen, die neben der Anlage in eben diese Versicherungen auch direkt in Wohnimmobilien investieren. Darin dokumentiert sich letztlich eine Responsibilisierung, im Sinne einer Aktivierung notwendig bürgerschaftlicher Verantwortlichkeit für soziale Zusammenhänge im Zuge neoliberalen Regierens (Lessenich 2008), indem Menschen nämlich mit gesicherter Kapitalanlage eine vorausschauend selbstverantwortliche Lebensführung beweisen. „In Bezug auf das Wohnen bedeutet dies, dass Individuen mehr und mehr dazu aufgerufen werden, Eigentum zu erwerben, um sich gegen Risiken des Alters abzusichern“(Heeg 2018, S. 52).

Mit diesen Prozessen von Deregulierung, Privatisierung und Finanzialisierung als veränderte resp. gemindert sozial-regulative Steuerungsweisen zeigt sich der Themenkomplex Wohnen immer weniger vom Bedarf und einer sozialstaatlichen Verantwortung her bestimmt. Hingegen wird Wohnen resp. Wohnraum zunehmend als Ware mit der Maxime zur Gewinnorientierung (einer industriekapitalistisch etablierten Marktlogik) verhandelt. Die Maxime neoliberaler Agenden, dass der Markt das Verhältnis von Angebot und Nachfrage regulieren würde, geht im Wohnen insbesondere dahingehend nicht auf, als hier Anleger*innen und Nutzer*innen mit unterschiedlichen Interessen agieren, und die Frage von Bedarf und Bedürfnissen der Wohnenden aber auch gesellschaftliche Implikationen weitgehend außenvorbleiben: „Insbesondere angesichts einer erstarkenden Tendenz der Finanzialisierung der Wohnungswirtschaft werden Wohnungen zunehmend gebaut und erworben, um Geld profitabel anzulegen. Die Erfüllung menschlicher und gesellschaftlicher Grundbedürfnisse ist hingegen deutlich in den Hintergrund getreten. Diese radikalisierte Dominanz des Tauschwerts über den Gebrauchswert gilt es umzukehren“ (Holm 2018, S. 147). Verstärkt wird dieser Eindruck dadurch, dass auch aktuelle Bautätigkeiten vielerorts dieser Direktive unterliegen, dass nämlich oftmals im hochpreisigen weil lukrativeren Segment gebaut wird, was am tatsächlichen Bedarf vorbeigeht. Dies trifft im konkreten Wohnalltag schließlich sozial polarisierend jene Menschen, die Wohnungen im unteren und mittleren Preissegment brauchen (Schönig 2018, S. 12), - oft

\footnotetext{
1 Der Bestand an gebundenen Mietwohnungen hat sich von ca. 4,0 Mio. Wohnungen in den 1980er Jahren auf ca. 1,2 Mio. im Jahr 2018 reduziert (Deutscher Bundestag 2019).
} 
„working poor“, einkommensschwache und/oder große Familien, Alleinerziehende, Geflüchtete u.a. Wohnpolitische Maßnahmen wie etwa Mietendeckel, Mietpreisbremse, Anpassung des Wohngeldes, neue Stärkung des sozialen Wohnungsbaus oder Baulanderschließung zur Schaffung neuen Wohnraums u. a. sollen hier gegensteuern. Dabei bleiben sie auf eine quantitativ ausreichende Wohnraumversorgung und deren Bezahlbarkeit fokussiert.

Ergänzend lohnt es jedoch, Wohnentwicklungen breiter zu betrachten, also nicht nur Markt- und Steuerungsmechanismen als Motoren von Veränderungen, sondern auch gesellschaftliche Entwicklungen und veränderte Lebensführungen daraufhin zu beleuchten, wie sie Wohnverhältnisse verändern. Auch gilt es zu berücksichtigen, welche weiteren Politiken und Diskurse hier als Schubkräfte wirken (aber auch, wer in einer solch gestalterischen Perspektive nicht sichtbar wird). Dies soll hier mit vier unterschiedlichen Schlaglichtern exemplarisch herausgestellt und nachgehend analytisch betrachtet werden:

Die Attraktivität des Urbanen Angespannte Wohnungsmärkte und daran geknüpfte Wohnproblematiken zeigen sich regional polarisiert vor allem in wachsenden Stadtregionen, Großstädten und auch kleineren Universitätsstädten, die Arbeits- und Ausbildungsplätze, aber auch Zentralitätsvorteile und Lebensqualität versprechen (Schönig 2018, S. 129). Sie wirken damit für mehrere Zielgruppen in der sich demographisch wandelnden Gesellschaft attraktiv und bewirken eine „Renaissance des Stadtwohnens“ (Kaltenbrunner und Waltersbacher 2014, S. 10): Neben Arbeitnehmenden der neuen Dienstleistungs- und Wissensgesellschaft, für die ein städtisches Milieu attraktiv ist (und durch Kommunen attraktiv gemacht wird), sieht Stadt- und Wohnsoziologe Walter Siebel Städte vor allem unter Zuwandernden begehrt - nicht nur aufgrund leichteren Arbeitszugangs, sondern auch einer erwartet größeren Toleranz für Fremde(s) und aufgrund bereits ansässiger Landsleute (Phänomen der Kettenwanderung; Siebel 2020, S. 15). Aber auch die verstärkte Arbeitsbeteiligung von Frauen und die darin liegende Abhängigkeit zu haushaltsnahen Dienstleistungen macht er als wesentlichen Mechanismus aus (ebd.). Städtische Kontexte werden dadurch nicht nur in ihrem Arbeits- und Teilhabe-, sondern auch in einem Vereinbarkeits- und Versorgungsversprechen sichtbar, wie dies auch Pohl (2010) für junge Familien herausarbeitet, und Gardemin als Grund für den neuerlich verstärkten Zuzug älterer Menschen ansieht. „Ziemlich zeitgleich zieht es zwei wesentliche Gruppen zurück in die Städte: Familien und Senioren. Sie profitieren von kurzen Wegen, Zugang zu Bildung, Kultur, Gesundheit und Konsum“ (Gardemin 2013, S. 25). (Re-)Urbanisierung und in der Folge auch innerstädtische Verdrängungsprozesse sind damit auch in einem breiten Streben nach Urbanität begründet resp. spätmodernes Leben auf Urbanität angewiesen (Siebel 2020). „Angesichts der Individualisierung bekommt die Wohnfunktion in der Stadt eine neue Bedeutung als Reurbanisierung" (Hannemann 2014, S. 42). Das Urbane steht dafür, wie sich hier Anforderungen aus individualisierten Lebensführungen (einfacher) bewältigen und gestalten lassen. So arbeiten Berding und Bukow Urbanität als „Narrativ“ heraus, das aktuell viele Quartiersentwicklungen prägt und ,die Verknüpfung von Arbeiten, Wohnen und Versorgung in einem praktikablen, alltagstauglichen und überschaubaren Lebensumfeld" verspricht (Berding und Bukow 2020). 
Wohnen im Alter Eine Angewiesenheit auf veränderte Wohnarrangements zeigt sich auch in neuen Wohnformaten, wie sie sich in den letzten Jahren vor allem in Kontexten des Alter(n)s zunehmend ausmachen lassen. Vorangetrieben durch Interessensverbände, soziale Institutionen der Altenhilfe, politische Programme, aber auch älter werdende Menschen selbst vollziehen sich in vielen Kommunen altersgerechte Quartier- und Stadtentwicklungen inkl. spezifisch unterstützter Wohnformen. ${ }^{2}$ Sie zielen - auch aus der erwiesen hohen Bedeutung des Wohnumfeldes für gelingendes Alter(n) (Naumann und Oswald 2020) - mit entsprechender Infrastruktur sowie sozialer und räumlicher Einbindung ins Gemeinwesen auf ein selbstbestimmtes Wohnen älterer Menschen, auf deren (auch aktivierungspolitisch gewollte) Mitwirkung, auf Versorgungssicherheit (unabhängig/er von Familie) sowie Lebensqualität und Teilhabe im Alter (van Rießen et al. 2015). Neuere Forschungsarbeiten betonen auch die wachsende Bedeutung von räumlich spezifischen Wohn- und Quartiersgestaltungen, wie sie einer wachsenden Zahl von Menschen mit Demenz bessere Alltagsorientierung und damit längere Selbstständigkeit bieten können (Wüstenrot Stiftung und Simon-Philipp 2020). Damit sind Demographie resp. die Alltagsbedürfnisse einer alternden Gesellschaft, insbesondere das Engagement und veränderte Wohnvorstellungen der sogenannten jungen Alten als ein wesentlicher Motor von Wohn- wie Stadtentwicklung zu sehen, die zunehmend neue Konzepte prägen (wie dies für andere Lebenslagen und -phasen, wie etwa Alleinerziehende, Zugewanderte u.a., auch wünschenswert wäre). Obgleich dadurch die grundsätzliche Frage des Wohnens und Versorgtseins im Alter für die Breite der Bevölkerung jenseits herkömmlicher (und für viele unzulänglichen) Heimunterbringungen insbesondere bei wachsender Unterstützungsbedürftigkeit kaum zufriedenstellend gelöst ist. ${ }^{3}$ Auch findet die Frage der wachsenden Wohnungsnot im Alter, die sich durch eine prognostiziert steigende Altersarmut, aber auch fehlende barrierefreie Wohnungen ergibt, kaum Eingang in die oben genannten Debatten einer prekären Wohnraumversorgung. Bereits als ,graue Wohnungsnot“ verhandelt (Pestel-Institut 2018) führe diese jedoch letztlich zu Verdrängungsprozessen, deren Folgen für Ältere angesichts der großen Bedeutung des gewohnten Wohnumfelds besonders gravierend einzuschätzen sind.

Gemeinschaftliche Wohnprojekte Als nur kleines, aber analytisch aufschlussreiches und durchaus wirkmächtiges Rädchen im Wohnwandel sind Gemeinschaftliche Wohnprojekte zu sehen. Mit vielfältiger Bewohnerschaft, (weitgehend) selbstorganisierten Organisationsweisen aber auch spezifischen Räumen und Bauweisen (Gemeinschaftsräume/-werkstätten/-gärten u. a.) erfahren sie seit der Jahrtausendwende und verstärkt seit 2010 wachsenden Zuspruch in Bevölkerung und Politik (Fedrowitz 2016). Subjektiv bedeutend rücken empirisch neben der Bezahlbarkeit und Wohn-

\footnotetext{
2 Dies reicht bis zur Verankerung von ,selbstorgansierten Wohngemeinschaften“ im Wohn- und TeilhabeGesetz (WTG), die sich u. a. über größere Selbstbestimmung, zentrale Lage, auch Einbezug zivilgesellschaftlicher Akteur*innen auszeichnen.

3 Dies steht völlig im Gegensatz zum ausdifferenzierten Angebot an Wohn- und Hilfsformen in Kontexten der Kinder- und Jugendhilfe und führt in Fachkreisen zu lauten Rufen nach alternativen, auch hybriden Lösungen zwischen ambulant und stationär sowie entsprechenden gesetzlichen Regelungen (z. B. Klie 2018).
} 
sicherheit, die über die Organisationsformen erreicht wird, wesentlich auch Fragen von Alltagstauglichkeit und Partizipationsmöglichkeiten vor dem Hintergrund sich wandelnder Lebensführungen in den Blick (Beck 2021). Sich raum-zeitlich und in kollektiven Settings ergebende Gelegenheiten in Wohnprojekten erleichtern etwa jungen Eltern Familie und Beruf zu vereinbaren, und dennoch auch Zeit für persönliche Kontakte zu haben. Diese und andere Zugewinne verweisen zugleich auf individuelle Bewältigungsherausforderungen und Verunsicherungen, die sich durch Entgrenzungsprozesse ergeben und sich auch im Wohnen niederschlagen - etwa in biographischen Übergängen, durch Fragen geschlechtergerechter Arbeitsteilung und Vereinbarkeit in Familien, aber auch in puncto Versorgungssicherheit und Teilhabe im Alter oder als Suche nach Selbstverortung. Die Notwendigkeit einer alltäglichen Lebensführung (Jurczyk et al. 2009), also Alltag individuell sinnvoll zu organisieren und zu bewältigen, wird hier als Gestaltungsaufgabe auch von Wohnen sichtbar. Gemeinschaftliches Wohnen lässt sich insofern in seinen (mehr oder weniger) bewussten, emanzipatorischen, gegenkulturellen Praktiken auch als Lebensbewältigung/ -gestaltung sehen, indem Wohnarrangements hier kollektiv, aber auch räumlich und strukturell anders gestaltet und darüber individuelle Alltagspraxen anders ermöglicht werden.

Empirisch zeigt sich hierfür bedeutend, flexible Räumlichkeiten individuell wie gemeinsam nutzen und (mit)gestalten zu können, strukturell mitentscheiden zu können, aber vor allem auch ein solidarisches Miteinander in den Projekten zu leben, worüber sich neue Erfahrungen (mit)bedingen. Generiert werden können dadurch unterschiedliche private, halböffentliche und öffentliche Sphären, worüber die Menschen neue Rollen und Arbeitsteilungen, neue Betätigungsmöglichkeiten nach innen und außen aber auch Sicherheiten für Alltagsgestaltungen, und darin letztlich subjektive Sicherheit und Handlungsfähigkeit gewinnen können. So können sich hier Ältere potenziell nicht nur als die aus dem Erwerbsleben Ausgeschiedenen erfahren, sondern auch als diejenigen, die etwa umfassende Projekterfahrung einbringen können, die sich um den gemeinsamen Garten kümmern und hierfür Wertschätzung erleben oder das Projekt nach außen vertreten o. Ä. Deutlich wird auch, wie hier (erst) in einem aktiven Aneignungsprozess strukturelle Anerkennung veränderter Lebensverhältnisse hergestellt und Resonanz (auch von außen) erfahren werden kann. Auch kann alternative Wohnkultur identitätsstiftend sein, Erfahrungen von Zugehörigkeit und Selbstwirksamkeit ermöglichen, was viele darin bestärkt, sich weiter und auch über das eigene Projekt hinaus zu engagieren. Entsprechend lassen sich Gemeinschaftliche Wohnprojekte als „Orte der Transformation“ (Beck 2020) diskutieren. Sie sind Resultat und Ausgangspunkt von Wohnveränderungen. Denn empirisch wird auch deutlich, wie Wohnprojekte im Zuge ihres Engagements im Umfeld, ihrer Vernetzung und Institutionalisierung wachsenden Zuspruch in Bevölkerung und Politik gewinnen (Pätzold 2019) und darüber auch Eingang in kommunale Strukturen und Planungsprozesse finden (etwa mit Ansprechpersonen in der Verwaltung, Beteiligung und Kontingente in Planungsverfahren u.a.). Was auch als Erfolg des oft langwierigen Engagements von zivilgesellschaftlichen Akteur*innen zu werten ist, steht damit zugleich in Gefahr, vereinnahmt und instrumentalisiert zu werden und zugleich zu Verdeckungen beizutragen. Zugespitzt gesagt, werden hier sozialstrukturelle Defizite von hochaktivierten Menschen (Lessenich 2008) eigenaktiv gelöst, was 
wiederum als Responsabilisierung zu diskutieren und im Impetus neosozialer Regierungsweisen unbedingt auch kritisch zu betrachten ist (Kessl 2005). „So schillert gemeinschaftliches Wohnen - im Modus von Aktivierung - eben auch als eigenaktive Lösung von strukturellen Ungewissheiten und Widersprüchen“ (Beck 2021, S. 351). Dadurch läuft Gemeinschaftliches Wohnen - ohne entsprechendes Gegensteuern - Gefahr, neuerlich jene Menschen sozial und räumlich auszuschließen, die nicht in der Lage oder gewillt sind, derart anspruchsvolle Projektentwicklungen zu leisten. Gerade diesem Aspekt wiederum versuchen Folgeprojekte zu entgegnen, indem sie auf die Notwendigkeit sozialer Strukturen verweisen und breitere Zugänge eröffnen wollen (Amann 2020).

Wohnproteste Nicht zuletzt zeigen sich in den letzten Jahren diverse Initiativen, Bündnisse, Bürgerforen etc., die dem derzeitigen Umgang mit Wohnraum aber auch weitergehenden gesellschaftlichen Entwicklungen widerständig begegnen. Als Bewegungen für ein Recht auf Wohnen und auf Stadt sind sie sich darin einig, dass sie sich für ,einen emanzipatorischen sozialen und politischen Wandel im Sinne einer ,Stadt für alle“ einsetzen“ (Vogelpohl et al. 2018, S. 105). Dazu lassen sich etwa Mieter*innen-Initiativen, Wohnprojekte, Quartiersinitiativen, auch Akteur*innen aus der Kulturbranche zählen, die mit unterschiedlichen Einmischungspraktiken, kreativen Aktionen und Protestkultur eine soziale Gestaltung von Wohnverhältnissen fordern und damit neue Wohn- und Stadtkultur (von unten) mit generieren. ,Diese Praktiken bilden einen Moment der Bewegung ab, die mehr einfordert als bezahlbaren Wohnraum“ (ebd., S. 116). Wie es über kollektiv widerständige Praktiken zumindest ansatzweise gelingen kann, ein kommunalpolitisches Umdenken zu bewirken und Wohnentwicklungen zu beeinflussen, zeigen Joscha Metzger und Sebastian Schipper (2018) anhand der Entwicklungen in Frankfurt und Hamburg auf, wo diese einen postneoliberalen Paradigmenwechsel in der Wohnungspolitik erkennen. Diese Bewegungen schließen daran an, was Susanne Frank vorsichtig und mit Fragezeichen als ein ,Symbol des Unbehagens an der neoliberalen Stadt- und Gesellschaftsentwicklung (?)“ bezeichnet und als eine neue Phase von Gentrifizierung ausmacht, indem hier Gentrifizierung ,als Negativfolie, als negativer gemeinsamer Bezugspunkt“ fungiert (Frank 2018, S. 97).

\section{Relevante Perspektiven für eine ganzheitliche Sicht auf Wohnen}

Die schlaglichtartigen Einblicke in aktuelle Aushandlungen im Wohnen verdeutlichen, dass sich die Analyse und zeitgemäße Lösung aktueller Wohnproblematiken resp. die Wohnungsfrage nicht einseitig auf die räumliche und strukturelle Ausgestaltung, also auf die Frage bzw. Gestaltung der quantitativen und bezahlbaren Wohnraumversorgung reduzieren lässt. Auch lässt sich gar nicht eindeutig bestimmen, von wem oder was aktuelle Entwicklungen im Wohnen ausgehen, wer oder was Akteur*innen/Mechanismen/Motoren für Wohnveränderungen sind. Wohnen ist entsprechend differenziert und zugleich prozesshafter zu betrachten, wie ich hier durch unterschiedliche Rahmungen zu systematisieren versuche. So ist Wohnen grundlegend mehrdimensional (Meuth 2017) sowie als ,ein Feld komplexer Inter- 
essensgeflechte“ und ,in mehrfacher Hinsicht von elementarer Bedeutung für Individuen, Gesellschaft und räumliche Entwicklung“ zu sehen (Schönig 2018, S. 11; auch Holm 2018). Wesentlich scheint, die Frage einer ausreichenden aber eben auch zeitgemäßen Wohnraumversorgung im Lichte gesellschaftlicher Entwicklungen aber auch heutigen Praktiken der Lebensgestaltung zu betrachten. Kurz: Mit der Wohnungsfrage müssten wir auch stärker die Wohnfrage stellen (Beck und Reutlinger 2019), und im Zuge dessen Wohnen als eine soziale Praxis in spezifisch räumlichstrukturellen Formaten auffassen, an der vielfältige Akteur*innen beteiligt sind. Dies werde ich im Folgenden in vier Punkten präzisieren und anschließend theoretisch fassen.

\subsection{Wohnen resp. Wohnwandel in seinem gesellschaftlichen Kontext betrachten}

Wohnen ist - das wird vor allem im historischen Rückblick deutlich - immer im gesellschaftlichen Gefüge zu sehen und zu verstehen: ,Eine Definition des Wohnens funktioniert also nur in einem spezifischen Bezugssystem“ (Siebel 2020, S. 8). Nicht nur, dass die Entwicklungen auf dem Wohnungsmarkt und die derzeitige Wohnungsnot ,als Ausdruck der Transformation des Wohlfahrtsstaats“ zu sehen sind (Schönig 2018, S. 13f.). Auch die Auswirkung soziodemographischer Entwicklungen auf Lebensgestaltungen sind fürs Wohnen zu beleuchten (Hannemann 2014). So konstituiert sich die Wohnungsfrage heute auch über vielfach entgrenzte Lebensführungen und -verläufe mit veränderten Bedarfen resp. sozialen Fragen. Kurz: Die Wohnweise ist viel grundlegender zu den (veränderten, heterogeneren) Lebensweisen ins Verhältnis zu setzen, wie dies Grundanliegen der Wohnsoziologie ist (Häußermann und Siebel 1996). Besonders im historischen Vergleich verdeutlicht diese, wie sich modernes Wohnen erst seit der Industrialisierung und mit Höhepunkt in den 1950/60er Jahren über die Normalitätsmuster von Normalfamilie und Normalerwerbsarbeit herausbildete und im idealtypischen Modell kleinfamilialen, bürgerlichen Wohnens stattfindet. Wohnen war/ist in diesem Format - mit Verdeckung der dort meist durch Frauen geleisteten Care-Arbeit - im Wesentlichen auf Intimität und Erholung reduziert. Dieses Modell verliert jedoch mit dem Aufweichen der Normalfamilie als Sozial- und Versorgungseinheit seine soziale und mit dem Aufbrechen des Normalerwerbsverlaufs seine ökonomische Basis (Siebel 2020). Das heißt, im Zuge der Entgrenzung von Arbeit und Leben stellt sich die Frage des Wohnens für viele neu und legt zugleich Anforderungen an Stadtentwicklung offen. So wird Wohnen zur individualisierten Gestaltungsaufgabe im Lebensverlauf (Beck 2021), über die das Verhältnis von (sich wandelnder) Arbeit und (sich wandelnden) Sozialbezügen, wie insbesondere auch (sich wandelnden) Sorgebezügen, mitgestaltet - bzw. umgekehrt Wohnen auch lebensphasenspezifisch in Relation zu diesen Aspekten immer wieder neu ausgerichtet werden muss. Im Vergleich zu vormals auch ausschließend wirkenden Leitbildern und Formaten liegen darin zwar neue Gestaltungsmöglichkeiten im Wohnen, in denen Menschen auch eigens Identität finden können und müssen (Lebensstil), aber ebenso Verunsicherungen und ungleiche Gestaltungschancen erfahren (aufgrund ökonomischer, sozialer, kultureller und gesundheitlicher Ressourcen). 


\subsection{Wohnen resp. Wohnwandel auch im subjektiven Lebenszusammenhang verstehen}

Für eine ganzheitliche Sicht auf Wohnen braucht es deshalb zugleich den Blick auf den subjektiven Lebenszusammenhang und den Lebensverlauf. Eine subjektorientierte Betrachtung kann - im Sinne einer Lebensbewältigungsperspektive (s. unten) dazu dienen, Herausforderungen und entsprechende Bedarfe im Wohnen zu erkennen. Es geht darum, die Menschen und ihre konkreten Bedürfnisse im Blick zu haben und Wohnen notwendig von individualisierten Lebensgestaltungen, biographischen Aushandlungen zu beleuchten, und damit eine ,soziale Blindheit“ einer marktorientierten Wohnungsversorgung (Holm 2018, S. 142) zu überwinden. In den Fokus rücken sodann Alltagsgestaltungen, aber auch Teilhabe- und Aneignungsmöglichkeiten im Wohnen, die bislang wenig beachtet werden. Hier gilt es, Wohnen divers, vor allem auch im Hinblick auf brüchiger gewordene Arbeits- und Lebensverläufe und daraus resultierende Übergänge zu beleuchten, auch daraufhin etwa, wie Geflüchtete ankommen und Zugang finden, oder wie Ältere in einer verlängerten nachberuflichen Phase weiter dazugehören können. Relevante Fragen wären sodann: Was braucht es zum Wohnen heute? Was macht Wohnen heute aus - als Alleinlebende, als Eltern, als Zugewanderte, im Alter, als Alleinerziehende, in Übergängen ...? Mithin wäre Wohnen als lebensphasen- und lebenslagenspezifische Bewältigungsaufgabe zu beleuchten, wie und worüber sich Menschen im Wohnen, individuell gelingend und zwar funktional, sozial und identitätsstiftend - verorten oder Wohnverhältnisse adäquat mitgestalten können.

\subsection{Wohnen resp. Wohnwandel als Prozess unter Berücksichtigung vielfältiger Akteur*innen und in seinem Begrenzungs- und Ermöglichungspotenzial fassen}

Wichtig scheint in der Debatte folglich, Wohnen vor dem Hintergrund sozialen Wandels nicht essentialistisch zu fassen, sondern als Prozess resp. Praxis, an dem unterschiedlichste Wohnakteur*innen diskursiv und praktisch mitwirken. Wohnverhältnisse sind sodann nicht nur zwischen einer strukturellen und einer räumlichen Dimension des Wohnens, sondern als Dreieck unter Einbezug einer alltagsweltlich sozialen Perspektive und der Wohnenden selbst als Handlungszusammenhang auch mit Blick auf Ein- und Ausschlüsse zu beleuchten. Das heißt de facto Wohnende in ihrer Vielfalt und mit unterschiedlicher Wirkungsmächtigkeit, aber auch zivilgesellschaftliche Akteur*innen - neben den bislang im Fokus stehenden Markt- und wohnpolitischen Akteur*innen - stärker als bislang mitzudenken, wie dies aktuell die Dynamiken von Wohnprotesten oder alternativen Wohnformen, letztlich aber auch erfahrene Ohnmacht und sichtbar werdende Wohnungsnöte nahelegen.

\subsection{Wohnen im Verhältnis zu Raumordnungen resp. Raumplanungen sehen}

Wohnen ist in seiner Bedeutung nicht allein als individuelle Wohnsituation (in einer Wohnung, in einem Haus) zu sehen, sondern auch dahingehend, welche Bezüge sich darüber sozusagen nach außen eröffnen lassen. Wichtig ist ein Bewusstsein 
dafür, ,wie Lebensbereiche, die im Zuge der Funktionalisierung in der Moderne für lange Zeit auch räumlich aufgespalten wurden (Arbeit, Bildung, Sorge, Freizeit, Kultur usw.) und nur durch normalisierende Muster des Normallebenslaufs sowie einhergehende, geschlechtsspezifische Rollen zusammengehalten wurden [...], nun individuell arrangiert werden (müssen)“ (Beck 2021, S. 344). Dies rückt insbesondere Fragen von Versorgung, Mobilität, Begegnung, Teilhabe stärker in den Fokus. Die mancherorts bis heute noch industriekapitalistisch geprägte Raumordnung, die Lebensbereiche funktional trennt, steht dabei offensichtlich auch im Widerspruch zu Alltagsgestaltung und Bedürfnissen in modernisierten Lebensführungen, zumindest für bestimmte Lebenslagen und -phasen, indem sie beispielsweise die Fragen von Integration (jenseits von Normalerwerbsarbeit) wie auch Teilhabe- und Care-Fragen als ein Sorgetragen im umfassenden Sinne (jenseits von bislang familiären, geschlechtsspezifischen Zuständigkeiten) offen lassen. ${ }^{4}$ Gelingendes Wohnen bedingt sich im Einzelfall insofern auch darüber, wie alltagstauglich und inklusiv Wohnen bzw. Wohnumfelder erfahren werden und welche Möglichkeiten es gibt, sich von dort aus zu versorgen, sich einbinden und einbringen zu können. Dies wirft die Frage nach adäquaten Bauweisen, relevanten Institutionen und weitergehenden Gelegenheiten auf bzw. danach, wie Wohnbau zwingend als Teil von Stadtplanung/ -entwicklung angesichts von Entgrenzungen stärker mit Blick auf alle Lebensbereiche gedacht werden muss. Insbesondere ländliche bzw. strukturschwache Regionen, die diesbezüglich besondere individuelle wie auch soziale Herausforderungen bergen, geraten dabei zunehmend ins Visier. Dabei ist als raumtheoretische Folie mitzuführen, dass Sozialraum nicht mit Nahraum/Quartier resp. einem Container gleichzusetzen ist (Kessl und Reutlinger 2007), sich stattdessen Handlungsvollzüge immer auch darüber hinaus erstrecken (können), aber auch, dass der Nahraum in bestimmten Lebensphasen und -situationen von hoher Bedeutung sein kann resp. Menschen angesichts von finanziellen, gesundheitlichen, sozialen Einschränkungen von dessen Konstitution abhängig sein können.

\section{Zur Chance eines sozial(räumlich)en Verständnisses von Wohnen}

Die hier geforderte ganzheitliche Sicht auf Wohnen, wie ich sie durch diese mehrfachen Rahmungen zu präzisieren versucht habe, impliziert, aktuelle Wohnentwicklungen in ihrem Zusammenspiel von ohnehin vielschichtigen Praktiken der Wohnraumversorgung - im Detail also wohnungspolitische Instrumente, Gesetzesveränderungen, ökonomische und Finanzmarktaspekte, aber auch bauliche Vorschriften, Planungsaspekten usw. - um den Blick auf heutige Praktiken der Lebensführung bzw. darin liegenden Anforderungen für das Wohnen zu ergänzen. Kurz: Wohnraumversorgung ist mit dem Leben heute und damit das Soziale mit dem Räumlichen zusammen zu denken und Wohnen insgesamt als Prozess unter Beteiligung vieler

\footnotetext{
4 Die funktionalistisch-getrennten Raumordnungen, wie sie sich im Zuge der Industrialisierung herausbildeten, kamen etwa in Arbeiten von Le Corbusier bis in die 1940er-Jahren besonders deutlich zum Ausdruck. Er unterschied vier Grundbedürfnisse von „Arbeit“, „Verkehr“, ,Wohnen“ und „Erholung“ (Hilpert 1978).
} 
Akteur*innen zu sehen. Dabei ist von einem relationalen Verhältnis auszugehen, dass die vorherrschenden Formate alltägliche Wohn- und Lebensweisen ermöglichen oder begrenzen (können), aber auch, dass alltägliche Wohn- und Lebensweisen Wohnformate bewahren, verändern und hervorbringen (können).

Theoretisch betrachtet lassen sich Wohnzusammenhänge sodann nicht als etwas rein Statisches, auch nicht als etwas Planbares, nicht als rein Gebautes und nicht als bloßes Tun von Wohnenden denken, sondern als soziale Praxis, die im Zusammenwirken unterschiedlichster Akteur*innen aus Politik, Stadtplanung, Architektur, zivilgesellschaftlichen Akteur*innen - auch der Sozialen Arbeit, wie ich noch ausführe - bis hin zu den Wohnenden selbst erhalten oder verändert wird. Dies entspricht einem praxeologischen Verständnis, welches das Soziale nicht als etwas dem Handeln Vorgelagertes, nicht als Gegebenes, sondern als etwas in beständigem Tun (,,doing“) Hergestelltes erachtet (etwa Reckwitz 2003). In Anlehnung an raumsoziologische Überlegungen (etwa Löw 2012) und Arbeiten zum Sozialraum (etwa Kessl und Reutlinger 2007; Reutlinger und Wigger 2010), die Raum und Räumlichkeit als Herstellungsprozess und darin gründenden Erfahrungen beleuchten, kann Wohnen somit als „sozial(räumlich)e Praxis“ gefasst werden (Beck 2021). In einer solchen sozial(räumlich)en oder auch raumreproduktionistischen Perspektive lässt sich für das Wohnen verdeutlichen, wie unterschiedlichste Praktiken ineinandergreifen und darüber (soziale) Entwicklung bedingen (Abb. 1).

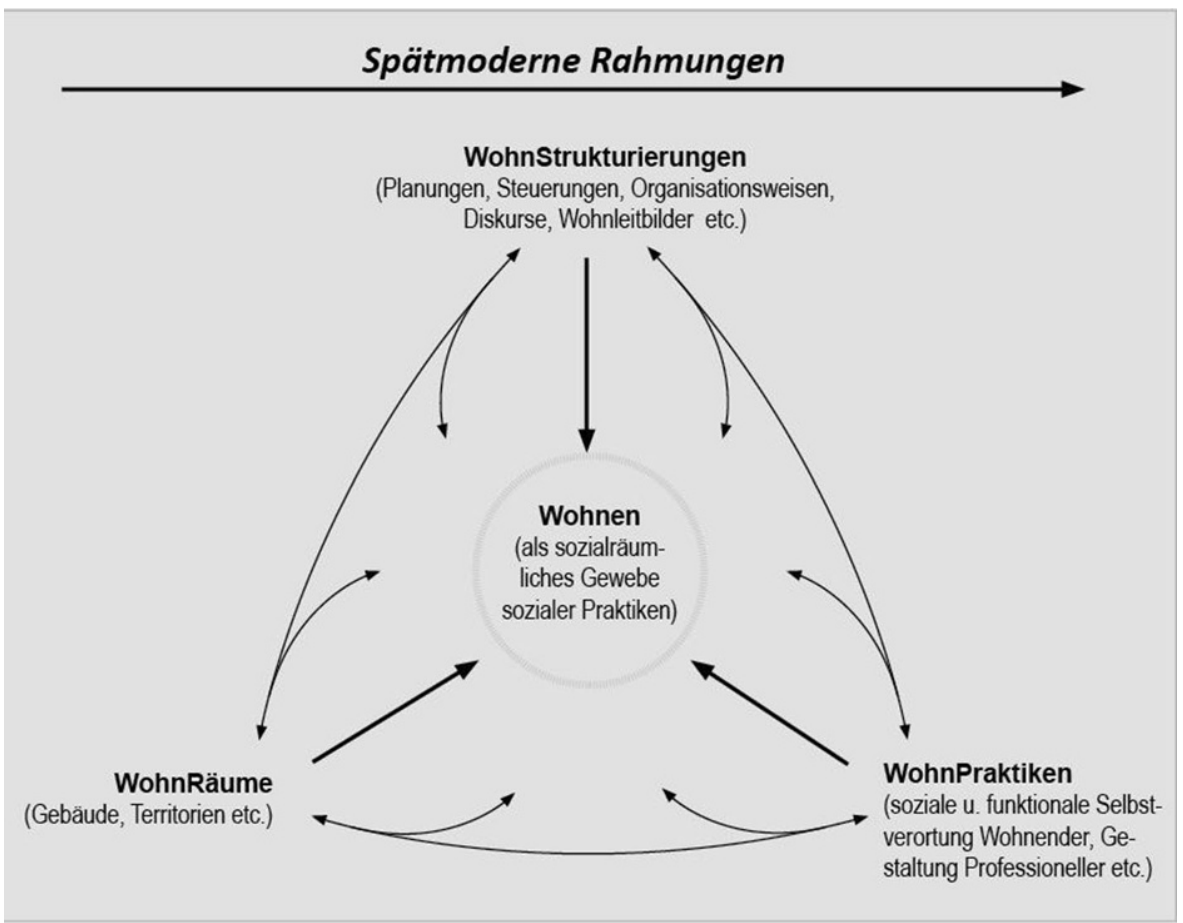

Abb. 1 „Wohn-Dreieck“ mit spätmodernen Rahmungen (Beck 2021; angelehnt an das St. Galler Modell des Sozialraums in Reutlinger und Wigger 2010) 
So ist Wohnen als sozial(räumlich)e Praxis immer im Zusammenwirken von Organisationsweisen, Gesetzgebungen, Programmatiken, auch Leitbildern (WohnStrukturierungen), von der Gestaltung von Orten und Gebäuden (WohnRäume) sowie von alltäglichen WohnPraktiken zu sehen, und dabei im Kontext gesellschaftlicher Entwicklungen und darin liegenden Ungleichheitsverhältnissen immer auch kritisch zu beleuchten. Wohnende sind hier als (mehr oder weniger wirkmächtige) Akteur*innen neben anderen Akteur*innen zu sehen, die Wohnverhältnisse (mit)(re)produzieren oder sich Veränderungen ohnmächtig ausgeliefert sehen. Gerade der Blick auf den (Re-)Produktionsprozess, also wie Wohnen von wem beständig oder veränderlich gestaltet wird, legt Ein- und Ausschlussmechanismen offen. Die Ökonomisierung des Wohnens als veränderte Strukturierung spielt dabei eine wesentliche und sehr wirkmächtige Rolle, es sind aber auch weitere Mechanismen zu berücksichtigen und weiter zu eruieren, auch wie sich Ungleichheitsverhältnisse darüber konstituieren oder verändern (können). Dazu gehören etwa der Emanzipationsprozess der Frau, der die bis dahin verdeckte Care-Frage nun auch als Wohnfrage offenlegt, ferner auch demographische Entwicklungen, Migrationsprozesse u. a., die veränderte Bedürfnisse im Wohnen und damit Anforderungen, wie Wohnräume und -umfelder konzipiert sein müssten, mit sich bringen. Gerade eine wachsende Selbstzuständigkeit, genauso aber auch eine wahrnehmbare „Kommunalisierung der Wohnungspolitik“ (Schönig 2018) verschließen dabei einen Blick auf übergeordnete gesellschaftliche Rahmenbedingungen als Erzeugungskontext. Entsprechend ist grundsätzlich einer Tendenz zu entgegnen, die soziale Probleme (allein) im Lokalen - hier im Wohnen - zu lösen versucht (zur Territorialisierung des Sozialen vgl. Kessl 2007).

Disziplinär gedacht, ist das Thema Wohnen folglich auch nicht nur mit Ökonomie, Wohnpolitik und Stadtplanung, sondern auch mit Sozialpolitik und schließlich - darauf will ich hinaus - auch Sozialer Arbeit zu denken.

\section{Zur Einmischungsnotwendigkeit Sozialer Arbeit}

Soziale Arbeit kann als Disziplin und Profession zu einer ganzheitlicheren Sicht auf Wohnen beitragen, weil sie um die konkreten Lebensbedingungen und alltäglichen Bewältigungsleistungen von Menschen weiß. Sie ist in lebensweltorientierter Sicht auf „den gegebenen Alltag ebenso wie auf den gelingenden Alltag bezogen“ (Thiersch 2020, S. 192) und hat damit ,im Aspekt der doppelten Anerkennung“ das Gegebene und Mögliche im Blick (ebd.): Sie unterstützt Menschen in individueller Alltagsgestaltung und Lebensbewältigung, arbeitet zugleich aber auch auf strukturelle Verbesserungen hin. Eine kritische Soziale Arbeit, wie sie angesichts ihres politischen Mandats fürs Wohnen relevant scheint, müsste zwangsläufig zu einer politischen Einmischung wie auch praktischen Mitgestaltung im Wohnen führen. „Kritik an und in den gegebenen Strukturen geht einher mit dem Entwurf besserer Möglichkeiten, die in den gegebenen Strukturen immer auch angelegt sind, die aber in der Arbeit an ihnen erkämpft und freigesetzt werden müssen“ (ebd., S. 193).

Was das Wohnen anbelangt, weiß Soziale Arbeit um die existenzielle wie auch alltägliche Bedeutung von Wohnen für gelingende Lebensgestaltungen. Sie sieht in ihrer praktischen Arbeit, wie sich Ungleichheitsverhältnisse im/über Wohnen ver- 
schärfen oder sich auch erst neu bilden. Die aktuell debattierten Wohnproblematiken dokumentieren sich in der Praxis der Sozialen Arbeit nicht nur in einer wachsenden Zahl an Wohnungslosen (BAG Wohnungslosenhilfe 2018), wahrnehmbar steigenden Existenznöten v. a. für Einkommensschwache, für Familien insbesondere von Alleinerziehenden, sondern auch in Diskriminierungserfahrungen auf dem Wohnungsmarkt etwa für Menschen mit Migrationshintergrund (Klus 2018), auch von Großfamilien u. a., oder in erfahrenen Stigmatisierungen, wenn Menschen schließlich in benachteiligt erachtete Stadtteile verdrängt werden. Eklatant aber wenig problematisiert zeigt sich außerdem, wie die in den letzten Jahren hinzugekommene Vielzahl an Geflüchteten vielerorts auf dem regulären Wohnungsmarkt (und damit auch in dieser Gesellschaft) nur schwer Fuß fassen können, obgleich dadurch die Debatte um Sozialen Wohnungsbau in der BRD erst forciert wurde (vgl. Kleilein und Meyer 2015).

Soziale Arbeit scheint ferner angesichts der Wohnungsmarktentwicklungen in vielen ihrer Arbeitsfelder - und das scheint empirisch dringend weiter auszuleuchten - in eigenen Einbindungsbemühungen eingeschränkt bis handlungsunfähig: etwa wenn übergangsmäßig gedachte kommunale Notwohnungen zur Dauerbleibe werden und sich darüber schwierige Lebenssituationen und Stigmatisierungen (mit)manifestieren, nur weil sich auf dem Wohnungsmarkt keine bezahlbare Wohnung finden lässt; oder wenn junge Erwachsene in schwierigen Lebenssituationen ihr Elternhaus oder auch Jugendhilfemaßnahmen verlassen wollen oder müssen und letztlich vermehrter auf der Straße landen, weil günstiger Wohnraum oder entsprechende Wohnkontingente fehlen (Specht 2018); auch wenn Resozialisierung nach Strafvollzug, oder ein Neubeginn für gewalterfahrene Frauen nach ihrem Aufenthalt im Frauenhaus für die Adressat*innen daran scheitern, dass für sie keine Wohnung auf dem Wohnungsmarkt zugänglich ist. Kurz: ,Wenn der Wohnungsmarkt so aussieht, dass sozial benachteiligte Leute keine Wohnung finden, dann ist die Soziale Arbeit an ihrer Grenze [...]. "5 Und letztlich bilden sich neue, bislang unbedarfte Adressat*innengruppen heraus, etwa wenn Familien in beengten Wohnverhältnissen, wachsenden Existenzsorgen und daraus hervorgehenden Konfliktdynamiken neu Unterstützung brauchen; oder wenn sich eine steigende Altersarmut mit steigenden Wohnungsmieten potenziert und zur Wohnungsnot führt. Die (unzureichende oder inadäquate) Wohnraumversorgung wird hier zu einem eigenständigen Ausschlussmechanismus, der die Frage aufwirft, wie dies auch und gerade durch Soziale Arbeit zu thematisieren und politisieren ist. Wie kann Soziale Arbeit Bewältigungsherausforderungen, auch Ausgrenzungserfahrungen und zugrundeliegende Mechanismen sichtbar machen und stärker auf sozialpolitischer Ebene platzieren? Wie kann sie sich für das Recht auf Wohnen, wie es als UN-Menschenrecht formuliert ist (Rausch 2011), einsetzen? Wie lassen sich auch Verteilungsfragen, Fragen sozialer Sicherung und Teilhabechancen, unbedingt aber auch (ungelöste) Care-Fragen mit Blick auf Wohnverhältnisse verdeutlichen, und eigene Unterstützungsangebote weiterentwickeln? Und welche räumlichen und strukturellen Gestaltungsnotwendigkeiten zeigen sich für Wohnarrangements, um den komplexer gewordenen Alltag

\footnotetext{
${ }^{5}$ Aktuelle Eindrücke aus Expert*innen-Interviews in verschiedenen Arbeitsfeldern im Rahmen von Praxiserkundungen mit Studierenden der Sozialen Arbeit an der Hochschule Esslingen.
} 
zu bewältigen? Wichtig scheint hier für die Soziale Arbeit zuvorderst auch einen eigenen Wohndiskurs zu führen, Wohnen im Lebenszusammenhang zu denken, und Wohnformate, nach wie vor gültige Leitbilder guten Wohnens, an denen auch sie mit ihren Unterstützungsangeboten festhält, zu hinterfragen.

Soziale Arbeit verfügt dabei über disziplinäre Sichtweisen, über die sich alltägliche Bedürfnisse und subjektive Bedeutungen im Wohnen erschließen lassen, auch welche Unsicherheiten und Chancen Menschen in ihrem Wohnzusammenhang erleben und worüber sie individuell Handlungsfähigkeit/-macht oder auch Diskriminierung/Ohnmacht erfahren (können). Konkret kann Soziale Arbeit über methodologische und methodische Zugänge der Lebensbewältigungsforschung (Böhnisch 2018; Litau et al. 2016) biographische Aushandlungsprozesse - im Verweis auf Agency-Konzepte auch mit Blick auf strukturelle und räumliche Begebenheiten beleuchten. Eine so verstandene subjektorientierte Wohnforschung (Beck 2021), wie sie auch der o.g. empirischen Arbeit zu Gemeinschaftlichem Wohnen zugrunde liegt, nimmt die Innensicht der Wohnenden ein und zeigt damit wie diese ihr Leben und darin liegende Bewältigungsherausforderungen auch im Wohnen biographisch aushandeln, auch wie Wohnpraxis dabei in bisherigen Formaten und Leitbildern reproduziert oder eigensinnig gebrochen wird. Problematische Wohnsituationen lassen sich dadurch in ihren begrenzenden Mechanismen beleuchten, gegenkulturelle Wohnpraktiken folglich als Bewältigungs- und Gestaltungsleistungen angesichts von Widersprüchen in herkömmlichen Wohnformaten lesen. Zugleich kann eine subjektorientierte Wohnforschung die Bedeutung von Aneignungsmöglichkeiten sichtbar machen, wie sie sich auch im Gemeinschaftlichen Wohnen vor allem darin zeigen, dass die Bewohner*innen über ihre eigenen Wohnverhältnisse mitentscheiden und Handlungszusammenhänge mitgestalten können. Sie kann erfassen, wie Erfahrungen von Mitwirkung das Gefühl bestärken kann, dazu zu gehören und sich weiter einbringen zu wollen, wie Bringfriede Scheu und Otger Autrata (2013) Partizipation in einer subjektorientierten Perspektive näher bestimmen. Ein praxeologisches Grundverständnis und eine subjektorientierte Perspektive auf Wohnen verdeutlichen somit das Wohnen machen resp. auch Stadt/Dorf machen (Können), worin mit der Lebensbewältigungsforschung auch potenziell (Selbst-)Bildungsprozesse auszumachen sind (Litau et al. 2016). Hierfür zeigen sich nicht zuletzt soziale Beziehungen, kollektive Settings für Prozesse der Ermächtigung relevant, aber auch Orte, die dies mitermöglichen, wie auch in der Bewegungsforschung und in der Sozialen Arbeit im Hinblick auf Empowermentprozesse und als wesentliche Gestaltungszugänge der Gemeinwesenarbeit diskutiert wird (Stövesand et al. 2013).

Zugleich begründet sich im Bewältigungsparadigma eine Einmischungsnotwendigkeit für die Soziale Arbeit. Auch wenn hierüber grundlegende sozialstaatliche Defizite nicht aufzuheben sind, bildet das eigene Wohnen und das Wohnumfeld für viele Menschen jenen Maßstab, worüber Alltag stattfindet und sie sich handlungsfähig und zugehörig erfahren können. Insofern kommt Sozialer Arbeit in einem interdisziplinären Diskurs wie auch praktisch ein wichtiger Gestaltungszugang zum Wohnen zu, den es stärker zu nutzen gilt (zu den Gestaltungszugängen Sozialer Räume vgl. das St. Galler Modell des Sozialraums, Reutlinger und Wigger 2010). 


\section{Möglicher Beitrag Sozialer Arbeit in der interdisziplinären Verhandlung und Gestaltung von Wohnverhältnissen ... und welche Chancen darin liegen}

Schon allein die Komplexität von Wohnen und Wohnfragen legt einen interdisziplinären Diskurs nahe (s. auch Schönig 2018). Dass sich Soziale Arbeit hierbei - und in der Folge auch in integrierten Planungs-/Gestaltungsprozessen - notwendig stärker beteiligt, lässt sich bereits mit der historischen Arbeitsteilung zwischen Sozialer Arbeit, Sozial-/Wohnpolitik und Stadtplanung/Wohnbau begründen. Erst in deren Zusammenwirken, zahlreichen Reformen und neuen Institutionen ließ sich letztlich die historische Soziale Frage und mit ihr die Wohnungsfrage stabilisieren (Beck und Reutlinger 2019). In einer raumreproduktionistischen Perspektive ist Soziale Arbeit entsprechend als Raumproduzentin zu sehen, die über ihr professionelles Handeln soziale wie auch räumliche Ordnungen (re)produziert (Dirks et al. 2016). Vor diesem Hintergrund wäre neben den inhärent wohn- und sozialpolitischen Fragen auch die sozialräumliche Gestaltung von Wohnverhältnissen unter Beteiligung Sozialer Arbeit - und somit die Gestaltung von Orten, die Gestaltung von Strukturen und die Arbeit mit Menschen (vgl. Reutlinger und Wigger 2010) in ihrem Zusammenwirken - zu beleuchten und mit der Idee sozial gerechter Gestaltung aufeinander abzustimmen. Zugleich braucht es einen kritischen Diskurs, um eingelagerte Ungleichverhältnisse und Responsibilisierungen aufzudecken. Die Chance, einer so umfassend gedachten interdisziplinären Sichtweise auf Wohnen liegt in einer relationalen Sicht, in der Strukturen und Räume des Wohnens in ihrer wechselwirkenden Bedeutung für soziale Prozesse aufgeschlossen werden können. Soziale Arbeit obliegt es, die Chancen einer sozialen Wohnraumversorgung hervorzuheben, die nicht nur Bezahlbarkeit, sondern auch soziale Bedürfnisse berücksichtigt und oftmals an den Rand gedrängte Bevölkerungsgruppen einschließt. Entsprechend ist Wohnen als soziale Infrastruktur (Holm 2014) weiterzudenken, welche heterogene Lebenslagen strukturell anerkennt, einer überhöhten Selbstzuständigkeit und daran geknüpften neuen Ausschlüssen entgegnet, ohne ermächtigende Kräfte von Selbstorganisation und Mitwirkung auszubremsen.

In der konkreten Ausgestaltung wäre erstens zu überlegen, wie Soziale Arbeit im Schulterschluss mit den aktuellen Sozialen (Wohn)Bewegungen resp. neuen sozialen Wohninitiativen, wie sie sich vielerorts etwa als Genossenschaften oder in anderen Formen zeigen, kooperativ agieren kann, so wie sie sich seit ihren Ursprüngen schon immer (in Auseinandersetzung) mit Sozialen Bewegungen und in Kontexten der Selbstorganisation (weiter)entwickelt hat (Wagner 2009). So plädiert auch Andrej Holm (2018) für eine stärkere Abstimmung von Sozial- und Wohnungspolitik, Quartier- und Infrastrukturplanung, und stellt letztlich auch infrage, inwieweit der Markt überhaupt eine sozial organisierte Wohnungsversorgung erfüllen kann. Hingegen ist Wohnungspolitik und Wohnraumversorgung ,als Teil einer lokalen Sozialen Daseinsvorsorge und Teil integrierter Stadtentwicklungspolitik zu betrachten“ (Schönig 2018, S. 20).

Neben solchen (gemeinwohlorientierten) Organisationsformen von Wohnraum, die durch Bezahlbarkeit, aber auch durch Mitbestimmungs- und Wohnrechte die Wohnsicherheit der Menschen stärken, wären - zweitens - gestaltbare Orte und so- 
ziale Infrastrukturen stärker in Wohnkontexten mitzudenken oder neu zu konzipieren, die Teilhabe ermöglichen, aber auch alltägliche Lebensführung unterstützen und bereichern. Zugleich wäre damit Stadt in ihrer funktional-kapitalistischen Raumordnung zu hinterfragen bzw. auch hinsichtlich Alltagstauglichkeit für alle Lebensalter und -formen neu zu denken (Feldtkeller 2012).

Drittens wären aber auch Gestaltungsprozesse an sich - und damit auch andere Konzepte von Stadtplanung/-entwicklung (aber auch Dorfentwicklung) - zu stärken, die Bewohner*innen ermöglichen, eigene Wohn- und Lebensverhältnisse mitzugestalten. Wesentlich zeigen sich hierfür integrierte Planungsperspektiven und kooperative Prozesse, wie sie sich im Verweis auf Governance bereits als neue Form von Quartiersentwicklung zeigen (Grzesiok 2018), als ,koproduktive Raumentwicklung“ diskutiert (Finkenberger und Schlaich 2014) oder auch als Verfahren von Sozialplanung (Böhmer 2015), speziell auch Altenhilfeplanung (Schubert 2019), weiterentwickelt werden. Hier stehen neue Zusammenarbeiten von Zivilgesellschaft, Wirtschaft und Kommunen, auch Fragen der Beteiligung im Zentrum und stützen sich auf ressortübergreifende Vorgehensweisen, indem bauliche, soziale und wohnstrukturelle Aspekte zusammen gedacht werden. Soziale Arbeit kann sich hier für ihre Adressat*innen einbringen bzw. diese dazu befähigen, sich einzubringen, hierfür geeignete Wege und Foren einfordern und darüber sozial gerechte(re) Wohnverhältnisse (mit)herstellen, wie sie dies vielerorts bereits tut.

Darin liegt zugleich eine doppelte Chance für die Soziale Arbeit: Zum einen könnte sie ihr bisheriges Bestreben lebensweltnaher Unterstützungsangebote, wie sie sich etwa in der Entwicklung der Gemeindenahen Psychiatrie, dezentralen Angeboten, Prävention, Alltagsnähe und Niedrigschwelligkeit abbilden, neu stärken. Wesentlich scheinen nahräumliche und breiter gedachte soziale Infrastrukturen, auch für eine alternative Arbeits-, Beteiligungs-, Sorge- und Wohnkultur, - im Sinne einer „Kultur des Sozialen zwischen Alltagssolidarität, Zivilgesellschaft und vernetzten Angeboten in neuen Kooperationen und neuen hybriden Organisationen" (Thiersch 2020, S. 12). Hier käme vor allem der Gemeinwesenarbeit eine größere Rolle zu, die ja schon immer auch Wohnthemen aufgreift, Einmischungspraktiken unterstützt, emanzipiert und konfliktorientiert auf gesellschaftliche Veränderungen ausgerichtet ist (Stövesand et al. 2013) und zugleich konkrete Partizipations-, Kultur- und Bildungsräume bietet, über die sich Wohnkultur und Alltag auch identitätsstiftend anders gestalten lässt.

Zum andern könnte sich Soziale Arbeit dadurch neu positionieren, indem sie sich in ihrem Selbstverständnis in einer Zuständigkeit für alle Lebenslagen und -alter inklusiver verortet. Dies bedeutet zum einen, weniger von der Zielgruppe der sogenannt Randständigen (sowie einer Problemsicht und Kritik des Verhaltens) auszugehen, wie Soziale Arbeit dies als Profession bisher in ihrer Fokussierung auf Bedürftige tut, sich aber angesichts des immanenten Ausschlusses kritisch hinterfragen muss (Anhorn et al. 2018). Vielmehr müsste sie inklusiv gedacht per se heterogene Lebenslagen im Blick haben und als Kritik der Verhältnisse solidarische Rahmungen fördern.

Hierfür ist es grundlegend hilfreich, Soziale Arbeit als Projekt zu sehen (Thole 2012), da sie sich beständig mit gesellschaftlichen Entwicklungen kritisch auseinandersetzt, sich reflexiv immer wieder überdenkt und - entlang ihrer Grundsätze von 
sozialer Gerechtigkeit, Bildung und Ermöglichung - in ihren Handlungsansätzen weiterentwickelt. Will Soziale Arbeit Wohnzusammenhänge und damit auch Stadtentwicklung mitgestalten, scheint ferner ein reflexiv-räumliches Verständnis wichtig (s. auch Fritsche und Wigger 2016), auch, dass sie ihre Rolle in der interdisziplinären Zusammenarbeit mit Akteur*innen von Wohn- und Stadtentwicklungen (neu) schärft, auch, indem sie sich etwa ihrer historischen Verknüpfungen bewusstwird (Oehler und Drilling 2016). Gewinnbringend wären außerdem weitere empirische Arbeiten, die insbesondere die Adressat*innenperspektive zum Vorschein bringen und erforderliche Wohnbedingungen für spezifische Lebenslagen (etwa Alleinerziehende, Zugewanderte, junge Erwachsene im Übergang u.a.) herausstellen - auch inwieweit ihnen räumliche und strukturelle Aspekte Handlungsspielräume eröffnen oder begrenzen. Und nicht zuletzt scheint eine selbstreflexive Haltung und Bewusstsein dafür bedeutend, für wen, was und wie Soziale Arbeit eintritt, weil sie darüber auch Adressierungen generiert, die neuerliche Fremd- und Selbstzuschreibungen mit sich bringen (Bitzan und Bolay 2017). Denn durch jede öffentliche Aktion und Thematisierung trägt Soziale Arbeit immer auch mit dazu bei, wie die Orte und deren Nutzer*innen wahrgenommen werden.

Funding Open Access funding enabled and organized by Projekt DEAL.

Open Access Dieser Artikel wird unter der Creative Commons Namensnennung 4.0 International Lizenz veröffentlicht, welche die Nutzung, Vervielfältigung, Bearbeitung, Verbreitung und Wiedergabe in jeglichem Medium und Format erlaubt, sofern Sie den/die ursprünglichen Autor(en) und die Quelle ordnungsgemäß nennen, einen Link zur Creative Commons Lizenz beifügen und angeben, ob Änderungen vorgenommen wurden.

Die in diesem Artikel enthaltenen Bilder und sonstiges Drittmaterial unterliegen ebenfalls der genannten Creative Commons Lizenz, sofern sich aus der Abbildungslegende nichts anderes ergibt. Sofern das betreffende Material nicht unter der genannten Creative Commons Lizenz steht und die betreffende Handlung nicht nach gesetzlichen Vorschriften erlaubt ist, ist für die oben aufgeführten Weiterverwendungen des Materials die Einwilligung des jeweiligen Rechteinhabers einzuholen.

Weitere Details zur Lizenz entnehmen Sie bitte der Lizenzinformation auf http://creativecommons.org/ licenses/by/4.0/deed.de.

\section{Literatur}

Amann, M. (2020). Mehr als Baugruppe und alternatives Wohnprojekt. Kommentar zu Lisa Vollmer Boris Michel „Wohnen in der Klimakrise. Die Wohnungsfrage als ökologische Frage“. Sub urban. Zeitschrift für kritische Stadtforschung, 8(1-2), 237-242.

Anhorn, R., Schimpf, E., Stehr, J., Rathgeb, K., Spindler, S., \& Keim, R. (Hrsg.). (2018). Politik der Verhältnisse - Politik des Verhaltens. Widersprüche der Gestaltung Sozialer Arbeit. Wiesbaden: Springer VS.

BAGW (2018). Handbuch der Hilfen in Wohnungsnotfällen. Entwicklung lokaler Hilfesysteme und lebenslagenbezogener Hilfeansätze (2. Aufl.). Berlin, Düsseldorf: BAG Wohnungslosenhilfe e. V..

Beck, S. (2020). Orte der Transformation? Gemeinschaftliche Wohnprojekte zwischen Widerständigkeit und Vereinnahmung. INDES - Zeitschrift für Politik und Gesellschaft, 2020(2), 78-86.

Beck, S. (2021). Wohnen als sozialräumliche Praxis. Zur subjektiven Bedeutung von Gemeinschaftlichem Wohnen im Kontext sozialen Wandels. Wiesbaden: Springer VS.

Beck, S., \& Reutlinger, C. (2019). Die Wiederkehr der Wohnungsfrage. Historische Bezüge und aktuelle Herausforderungen für die Soziale Arbeit. Zürich: Seismo. 
Berding, N., \& Bukow, W.-D. (Hrsg.). (2020). Die Zukunft gehört dem urbanen Quartier. Das Quartier als eine alles umfassende kleinste Einheit von Stadtgesellschaft. Wiesbaden: Springer VS.

Bitzan, M., \& Bolay, E. (2017). Soziale Arbeit - die Adressatinnen und Adressaten. Opladen, Toronto: Barbara Budrich.

Böhmer, A. (2015). Verfahren und Handlungsfelder der Sozialplanung. Grundwissen für die Soziale Arbeit. Wiesbaden: Springer VS.

Böhnisch, L. (2018). Sozialpädagogik der Lebensalter. Eine Einführung (8. Aufl.). Weinheim: Beltz Juventa.

Deutscher Bundestag (2019). Wohnungspolitische Bilanz der Bundesrepublik Deutschland seit 2006. Drucksache, Bd. 19/12786. Köln: Bundesanzeiger Verlag GmbH.

Dirks, S., Kessl, F., Lippelt, M., \& Wienand, C. (2016). Urbane Raum(re)produktion - Soziale Arbeit macht Stadt. Münster: Westfälisches Dampfboot.

Fedrowitz, M. (2016). Gemeinschaftliches Wohnen - Stand und Entwicklung in Deutschland. Nachrichten $\operatorname{der}$ ARL - Magazin der Akademie für Raumforschung und Landesplanung, 46(1), 9-12.

Feldtkeller, Andreas (2012). Zur Alltagstauglichkeit unserer Städte. Wechselwirkungen zwischen Städtebau und alltäglichem Handeln. Berlin, Tübingen: Hans Schiler.

Finkenberger, I., \& Schlaich, C. (2014). Zusammenleben in integrierten Nachbarschaften. APuZ - Aus Politik und Zeitgeschichte, 64(20-21), 46-52.

Frank, S. (2018). Gentrifizierung und neue Mittelschichten: Drei Phasen eines wechselhaften Verhältnisses. In B. Schönig, et al. (Hrsg.), Wohnraum für alle?! (S. 87-100). Bonn: bpb.

Fritsche, C., \& Wigger, A. (2016). Soziale Arbeit und Stadtentwicklung aus reflexiv räumlicher Perspektive. In M. Drilling \& P. Oehler (Hrsg.), Soziale Arbeit und Stadtentwicklung. Forschungsperspektiven, Handlungsfelder, Herausforderungen (2. Aufl. S. 71-85). Wiesbaden: Springer VS.

Gardemin, D. (2013). Großstadt im Wandel - Plädoyer für eine neue Wohnungspolitik. spw - Zeitschrift für sozialistische Politik und Wirtschaft, 5(198), 23-28.

Grzesiok, S. (2018). Bündnisse für Wohnen im Quartier. Ein Format integrierter und kooperativer Quartiersentwicklung. Wiesbaden: Springer VS.

Hannemann, C. (2014). Zum Wandel des Wohnens. APuZ - Aus Politik und Zeitgeschichte, 64(20), 36-43.

Häußermann, H., \& Siebel, W. (1996). Soziologie des Wohnens. Eine Einführung in Wandel und Ausdifferenzierung des Wohnens. Weinheim, München: Juventa.

Heeg, S. (2018). Finanzialisierung und Responsibilisierung - Zur Vermarktlichung der Stadtentwicklung. In B. Schönig, et al. (Hrsg.), Wohnraum für alle?! (S. 47-59). Bonn: bpb.

Hilpert, T. (1978). Die funktionelle Stadt. Le Corbusiers Stadtvision; Bedingungen, Motive, Hintergründe. Braunschweig: Vieweg.

Holm, A. (2014). Wiederkehr der Wohnungsfrage. APuZ - Aus Politik und Zeitgeschichte, 64(20-21), 25-30.

Holm, A. (2018). „Neue Gemeinnützigkeit“ und soziale Wohnungsversorgung. In B. Schönig, et al. (Hrsg.), Wohnraum für alle?! (S. 135-151). Bonn: bpb.

Jurczyk, K., Schier, M., Szymenderski, P., Lange, A. \& Voß, Günter G. (2009). Entgrenzte Arbeit - entgrenzte Familie. Grenzmanagement im Alltag als neue Herausforderung. Berlin: edition sigma.

Kaltenbrunner, R. \& Waltersbacher, M. (2014). Wohnsituation in Deutschland. APuZ - Aus Politik und Zeitgeschichte, 64(20), 3-12.

Kessl, F. (2005). Der Gebrauch der eigenen Kräfte. Eine Gouvernementalität Sozialer Arbeit. Weinheim, München: Juventa.

Kessl, F. (2007). Territorialisierung des Sozialen. Regieren über soziale Nahräume. Opladen: Barbara Budrich.

Kessl, F., \& Reutlinger, C. (2007). Sozialraum. Eine Einführung. Wiesbaden: VS.

Kleilein, D., \& Meyer, F. (2015). Flüchtlingsfrage? Wohnungsfrage! http://bauwelt.de/dl/974821/artikel. pdf. Zugegriffen: 19. Mai 2021.

Klie, T. (2018). Sorge und Pflege in Sozialräumen - Stärkung der kommunalen Verantwortung. Strukturreform Pflege und Teilhabe und die Differenzierung zwischen Care und Cure. In C. Bleck, et al. (Hrsg.), Alter und Pflege im Sozialraum. Wiesbaden: Springer.

Klus, S. (2018). Wohnen als Handlungsfeld der Sozialen Arbeit in der Migrationsgesellschaft. In B. Blank, S. Gögercin, K. E. Sauer \& B. Schramkowski (Hrsg.), Soziale Arbeit in der Migrationsgesellschaft (S. 723-733). Wiesbaden: Springer VS.

Lessenich, S. (2008). Die Neuerfindung des Sozialen. Der Sozialstaat im flexiblen Kapitalismus. Bielefeld: transcript.

Litau, J., Walther, A., Warth, A., \& Wey, S. (Hrsg.). (2016). Theorie und Forschung zur Lebensbewältigung. Methodologische Vergewisserungen und empirische Befunde. Weinheim, Basel: Beltz Juventa. 
Löw, M. (2012). Raumsoziologie. 7. Aufl. Frankfurt a. M.: Surhkamp.

Metzger, J., \& Schipper, S. (2018). Postneoliberale Strategien für bezahlbaren Wohnraum? Aktuelle wohnungspolitische Ansätze in Frankfurt am Main und Hamburg. In B. Schönig, et al. (Hrsg.), Wohnraum für alle?! (S. 181-212). Bonn: bpb.

Meuth, M. (2017). Theoretische Perspektiven auf Wohnen. Ein mehrdimensionales Wohnverständnis in erziehungswissenschaftlicher Absicht. In M. Meuth (Hrsg.), Wohn-Räume und pädagogische Orte. Erziehungswissenschaftliche Zugänge zum Wohnen (S. 97-122). Wiesbaden: Springer VS.

Naumann, D., \& Oswald, F. (2020). Wohnen im Alter. In K. Aner \& U. Karl (Hrsg.), Handbuch Soziale Arbeit und Alter (S. 369-377). Wiesbaden: Springer VS.

Oehler, P., \& Drilling, M. (2016). Soziale Arbeit, Gemeinwesenarbeit und Stadtentwicklung. Eine theoriegeschichtliche Spurensuche. In M. Drilling \& P. Oehler (Hrsg.), Soziale Arbeit und Stadtentwicklung. Forschungsperspektiven, Handlungsfelder, Herausforderungen (2. Aufl. S. 13-41). Wiesbaden: Springer VS.

Pätzold, R. (2019). Gemeinschaftliche Wohnformen. In Bundeszentrale für politische Bildung (Hrsg.), Gesucht! Gefunden? Alte und neue Wohnungsfrage (S. 175-187). Bonn: bpb.

Pestel-Institut (2018). Wohnen der Altersgruppe 65plus. Hannover: Pestel-Institut.

Pohl, T. (2010). Reurbanisierung als Trend? Folgen des Wandels der raumzeitlichen Alltagsorganisation in Familienhaushalten. In D. Läpple, U. Mückenberger \& J. Oßenbrügge (Hrsg.), Zeiten und Räume der Stadt. Theorie und Praxis (S. 45-64). Opladen, Farmington Hills: Barbara Budrich.

Rausch, G. (2011). Mensch kann nicht Nichtwohnen. WOHNEN - Ware oder Menschenrecht? In S. Elsen (Hrsg.), Ökosoziale Transformation. Solidarische Ökonomie und die Gestaltung des Gemeinwesens. Perspektiven und Ansätze der ökosozialen Transformation von unten (S. 235-268). Neu-Ulm: AG SPAK Bücher.

Reckwitz, A. (2003). Grundelemente einer Theorie sozialer Praktiken. Eine sozialtheoretische Perspektive. Zeitschrift für Soziologie der Erziehung und Sozialisation, 32(4), 282-301.

Reutlinger, C., \& Wigger, A. (Hrsg.). (2010). Transdisziplinäre Sozialraumarbeit. Grundlegungen und Perspektiven des St. Galler Modells zur Gestaltung des Sozialraums. Berlin: Frank \& Timme.

van Rießen, A., Bleck, C., \& Knopp, R. (Hrsg.). (2015). Sozialer Raum und Alter(n). Zugänge, Verläufe und Übergänge sozialräumlicher Handlungsforschung. Wiesbaden: Springer VS.

Scheu, B., \& Autrata, O. (2013). Partizipation und Soziale Arbeit. Einflussnahme auf das subjektive Ganze. Wiesbaden: Springer VS.

Schönig, B. (2013). Die neue Wohnungsfrage. Blätter für deutsche und internationale Politik, 2, 17-20.

Schönig, B. (2018). Sechs Thesen zur wieder mal ,neuen“ Wohnungsfrage - Plädoyer für ein interdisziplinäres Gespräch. In B. Schönig, et al. (Hrsg.), Wohnraum für alle?! (S. 11-26). Bonn: bpb.

Schubert, H. (Hrsg.). (2019). Integrierte Sozialplanung für die Versorgung im Alter. Grundlagen, Bausteine, Praxisbeispiele. Wiesbaden: Springer.

Siebel, W. (2020). „Und dann, 1969 im Frankfurter Westend, hat eine Hauseigentümerin ein Haus aus dem 19. Jahrhundert rosa angestrichen. Und das wurde zu einer Ikone.“ Ein Gespräch über das Phänomen des Wohnens. INDES - Zeitschrift für Politik und Gesellschaft, 10(2), 7-26.

Wüstenrot Stiftung, \& Simon-Philip, C. (Hrsg.). (2020). Vergessen in der Stadt. Stadtgestaltung von und für Menschen mit Demenz. Stuttgart: HFT Stuttgart.

Specht, T. (2018). Heranwachsende und junge Erwachsene. In BAGW (Hrsg.), Handbuch der Hilfen in Wohnungsnotfällen. Entwicklung lokaler Hilfesysteme und lebenslagenbezogener Hilfeansätze (2. Aufl. S. 347-369). Berlin, Düsseldorf: BAG Wohnungslosenhilfe e. V.

Stövesand, S., Stoik, C., \& Troxler, U. (Hrsg.). (2013). Handbuch Gemeinwesenarbeit. Opladen, Berlin, Toronto: Barbara Budrich.

Thiersch, H. (2020). Lebensweltorientierte Soziale Arbeit-revisited. Weinheim, Basel: Beltz Juventa.

Thole, W. (2012). Die Soziale Arbeit - Praxis, Theorie, Forschung und Ausbildung. Versuch einer Standortbestimmung. In W. Thole (Hrsg.), Grundriss Soziale Arbeit (S. 19-79). Wiesbaden: Springer VS.

Vogelpohl, A., Vollmer, L., Vittu, E., \& Brecht, N. (2018). Die Repolitisierung des Wohnens. Städtische soziale Bewegungen für ein Recht auf Wohnen und auf Stadt in Hamburg, Berlin, Jena und Leipzig. In B. Schönig, et al. (Hrsg.), Wohnraum für alle?! (S. 105-130). Bonn: bpb.

Wagner, L. (Hrsg.). (2009). Soziale Arbeit und Soziale Bewegungen. Wiesbaden: VS. 